\title{
An immunological view of chemotherapy
}

\author{
"With means to raise antitumor immunity before tumor resection, \\ such as chemotherapy plus IL-12, more postsurgery recurrent disease \\ can be prevented and better outcome and survival may be possible."
}

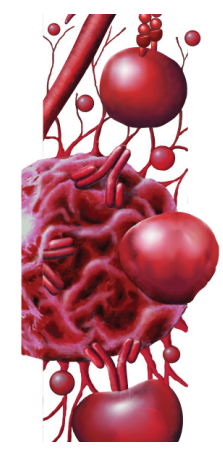

Keywords: antitumor immunity $\bullet$ autoimmunity $\bullet$ chemotherapy $\bullet$ CTLA-4-Ig $\bullet$ DAMP

- foreign antigen •IL-12・ metastases $\bullet$ PD1/PDL1 • Treg

No one is questioning the fact that chemotherapy can kill tumor cells by direct drug toxicity. What is being argued here is that not all-of-the efficacy of chemotherapy comes from this direct tumor cell killing. In hosts possessing pre-existing antitumor immunity against a primary tumor, chemotherapy activates this antitumor immunity through synchronized killing and antigen release from the primary tumor and the activated antitumor immunity contributes significantly (often predominantly) to the efficacy. This view, supported by some historical [1-3] and recent studies [4,5] may explain clinical observations in some cancer patients. For example, if direct toxicity by the chemotherapy drug alone is totally responsible for its efficacy, one would expect equal efficacy against equal tumor burdens regardless if primary or metastatic. But the clinical experience is that chemotherapy is more likely to be effective against stage II and III tumors than stage IV tumors. Further, clinical evidence suggests that chemotherapy is more effective against primary tumors with or without metastases than postsurgery metastases without a primary tumor. From the immune point of view, this may be explained by the presence and absence of pre-existing antitumor immunity. Antitumor immunity is a critical factor influencing tumor stage and controlling metastases [6]. This observation is consistent with findings from murine tumor models showing that the presence of preexisting antitumor immunity affects tumor response to chemotherapy profoundly [5]. Because antitumor immunity contributes so much to the antitumor responses of chemotherapy, it is suspected that loss of this immunity during continued therapy has a negative effect on antitumor responses. A common clinical observation is that chemotherapy, even when effective, does not completely eradicate tumor burden and subsequent repeat cycles of the same or even different drugs are less efficacious. Consistent with this acquired drug resistance, animal study shows that antitumor immunity is activated strongly during the initial cycle of chemotherapy but becomes less or inactivated during subsequent cycles of treatment. This acquired drug resistance may not be simply due to selection of drug resistant tumor cells as the mainstream dogma suggests because re-implanting tumor cells from mice demonstrating drug resistance into naive hosts returns full antitumor responses to the same chemotherapy [7]. From the immune point of view, this may be explained by the loss of activation/ participation by antitumor immunity that contributed significantly during the initial cycle of chemotherapy treatment. Indeed, one can observe a stepwise loss of participation by antitumor immunity associated with the loss of efficacy in animal tumor models [7]. The problem is that this type of 'one-time' immune response is common not only to chemotherapy, but also to other cancer therapies (an indication that immune participation may be important in many anticancer treatments). Suppression of antitumor immunity has been used to explain some of the cases, but even with therapies targeted to relieve such suppres-

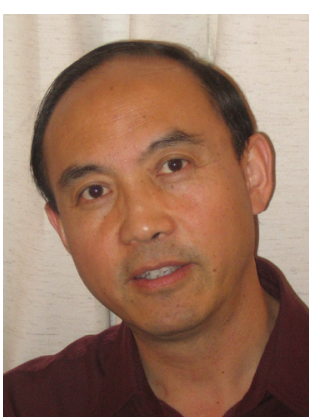

Kangla Tsung

Department of Surgery, Stanford University School of Medicine, Stanford, CA 94305-5641, USA

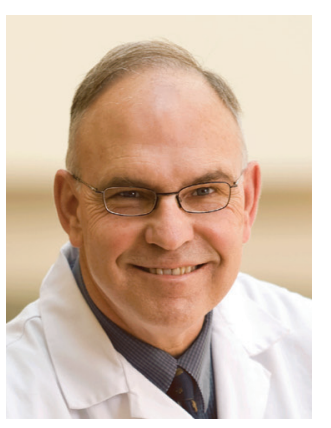

Jeffrey A Norton Author for correspondence: Department of Surgery, Stanford University School of Medicine, Stanford, CA 94305-5641, USA janorton@stanford.edu 
sion, for example, blockade of 'checkpoint' inhibitors (CTLA-4, PD1/PDL1), antitumor responses are rarely sustained long enough to achieve complete tumor eradication. Why is it that an initial response strong enough to cause apparent tumor regression cannot go to complete tumor eradication even with continued treatment? What happens to the activated antitumor immunity? Other than being inhibited or suppressed, antitumor immunity may become exhausted or even inhibited by antigen-specific immune tolerance [8]. If priming of the initial antitumor immune response is a special event that cannot be reproduced by later tumor development or therapy (in animal studies, injection of suspended tumor cells may constitute an immune boosting event [9]), it is possible that activation and use of primed antitumor $\mathrm{T}$ cells becomes a one-time event [10]. This would explain why removing immune inhibition by CTLA-4-Ig or anti-PD1/PDL1 cannot sustain its efficacy because these treatments may depend on activation of primed antitumor $T$ cells [11], or pre-existing antitumor immunity, and lose effect when these primed $\mathrm{T}$ cells are depleted. In another worse case possibility, antitumor immunity by nature of an auto-immunity may become tolerated. Studies in infection and auto-immunity have analyzed the fate of immunity against overwhelming antigens in the absence of clear distinction between self and nonself [12]. In essence, tumor is self and tumor antigens cannot be recognized as 'foreign' based on sequence alone. In the absence of other 'danger' signals (like the damage-associated molecular patterns [DAMP] associated with infection, for example), antitumor immunity will have no clear guidance toward continued amplification, deviation and attack. This lack of guidance may lead to recognition of the antigens being 'self' and further immune activation is terminated. In our experiments, we have seen this phenomenon by transferring spleen cells from mice bearing relapsed chemotherapy-resistant tumors into untreated tumorbearing mice and comparing responses to chemotherapy with mice receiving other control spleen cells. Different from exhaustion, tolerance is an active process and is dominant over activation. Consistent with this finding, supply mice bearing relapsed tumors from previous chemotherapy with spleen cells from tumorimmune donors did not reverse the resistance to chemotherapy, whereas the same tumor-immune cells transferred into normal untreated tumor-bearing mice enhance responses to subsequent chemotherapy [5]. These findings raise serious questions about improving chemotherapy efficacy from a immune point of view: if activation and participation of antitumor immunity account for most of chemotherapy efficacy, we need to find ways to preserve antitumor immunity during chemotherapy in order to achieve better efficacy. That is where IL-12 comes in.

"...with IL-12 added to chemotherapy, we have seen individual cases where a smaller tumor not responding to the initial cycle of chemotherapy treatment is eradicated by subsequent repeat cycles of chemotherapy in the presence of IL-12 when the tumor burden is actually larger."

IL-12 as a typical DAMP-induced danger cytokine was shown by many including us to be one of the best immune factors possessing strong antitumor activity in animal studies. Some early experiments demonstrating the power of IL-12-mediated antitumor responses are still the best examples what antitumor immunity can do under the right conditions [13]. But not all tumors respond to IL-12 therapy equally. Analyses made on the mechanism of IL-12-mediated antitumor response have indicated that IL-12-mediated antitumor immune responses depend on the presence of pre-existing antitumor immunity [14]. This is consistent with the profiles of IL-12 receptor expression on antigen-specific T cells being transient upon activation [15]. While chemotherapy provides the initial activation of tumor-specific $\mathrm{T}$ cells (and IL-12 receptor expression), IL-12 given at the right time serves as a signal to educate the $\mathrm{T}$ cells that the antigens they see are likely 'foreign' because only invading microbes induce IL-12 naturally. T cell responses thus modified will likely persist for longer periods of time [16] and resist down regulation by Treg and cytokines [17]. This is the explanation for the powerful antitumor efficacy we have observed previously and the reason why IL-12 is able to propel continued responses to chemotherapy in cases where an initial single treatment cycle did not eradicate the tumor completely [7]. Indeed, with IL-12 added to chemotherapy, we have seen individual cases where a smaller tumor not responding to the initial cycle of chemotherapy treatment is eradicated by subsequent repeat cycles of chemotherapy in the presence of IL-12 when the tumor burden is actually larger. This paradoxical observation indicates that volume of the tumor burden is not a limiting factor but rather the strength and persistence of antitumor immunity. It also suggests that the use of IL-12 can amplify pre-existing antitumor immunity activated by the previous cycle of chemotherapy to a higher level and avoid the common exhaustion/tolerance problem of chemotherapyactivated antitumor immunity in the absence of IL-12. Interestingly, experiments designed to measure this elevation of immunity demonstrated another even more significant use of IL-12. Tumor-bearing mice untreated or treated with chemotherapy or chemotherapy plus IL-12 underwent tumor resection to preserve the residual 
antitumor immunity measured by challenge with different doses of tumor cells after complete resection. The results demonstrated three clinically relevant phenomena. Among similar tumor-bearing hosts, the residual antitumor immunity following tumor resection varied widely from barely detectable to relatively strong when compared with naive mice. This wide variation of residual antitumor immunity is highly consistent from one experiment to another, indicating that it is a rule rather than exception that apparently similar tumor-bearing hosts may possess widely varied concomitant antitumor immunity that serves as the starting base for many subsequent immune responses such as the one activated by chemotherapy. Chemotherapy treatment, even though not curative, can lead to a clear elevation of postresection antitumor immunity. This is a mimic of neo-adjuvant chemotherapy in cancer patients. An immune mechanism may explain the observed improvement in longterm protection against recurrence and metastases when using neo-adjuvant chemotherapy in cancer patients. IL-12 added to chemotherapy elevates the postresection antitumor immunity to a very strong level often allowing thus treated mice to resist the highest dose of tumor

\section{References}

1 Ferrer JF, Mihich E. Dependence of the regression of sarcoma 180 in vitamin B 6-deficient mice upon the immunologic competence of the host. Cancer Res. 27(3), 456-461 (1967).

2 Moore M, Williams DE. Contribution of host immunity to cyclophosphamide therapy of a chemically-induced murine sarcoma. Int. J. Cancer 11(2), 358-368 (1973).

3 Radov LA, Korn JH, Haskill JS. Host immune potentiation of drug responses to a murine mammary adenocarcinoma. II. Effect of melphalan therapy on the host immune system. Int. J. Cancer 18(5), 630-638 (1976).

4 Apetoh L, Ghiringhelli F, Tesniere A et al. Toll-like receptor 4-dependent contribution of the immune system to anticancer chemotherapy and radiotherapy. Nat. Med. 13(9), 1050-1059 (2007).

5 Zhang L, Feng D, Yu LX et al. Preexisting antitumor immunity augments the antitumor effects of chemotherapy. Cancer Immunol. Immunother. 62(6), 1061-1071 (2013).

6 Ostrand-Rosenberg S, Grusby MJ, Clements VK. Cutting edge: STAT6-deficient mice have enhanced tumor immunity to primary and metastatic mammary carcinoma. J. Immunol. 165(11), 6015-6019 (2000).

7 Zhang L, Feng D, Hu Y et al. IL-12 augments antitumor responses to cycled chemotherapy. J. Immunother. 38(4), 137-144 (2015).

8 Huang X, Yang Y. The fate of effector CD8 T cells in vivo is controlled by the duration of antigen stimulation. Immunology 118(3), 361-371 (2006).

9 Ochsenbein AF, Klenerman P, Karrer U et al. Immune surveillance against a solid tumor fails because of rechallenge $\left(5-10 \times 10^{5}\right.$ cells $)$. Also, unlike the other treatments, this elevation of postresection antitumor immunity by IL-12-aided chemotherapy affects majority $(>70 \%)$ of treated hosts to demonstrate strong immune protection. This points out a way to improve the potential benefit of neo-adjuvant chemotherapy. Inasmuch as tumor burden reduction is concerned, surgery remains the best choice. It is often the postsurgery recurrence and metastases that affect survival following tumor resection. With means to raise antitumor immunity before tumor resection, such as chemotherapy plus IL-12, more postsurgery recurrent disease can be prevented and better outcome and survival may be possible.

\section{Financial \& competing interests disclosure}

The authors have no relevant affiliations or financial involvement with any organization or entity with a financial interest in or financial conflict with the subject matter or materials discussed in the manuscript. This includes employment, consultancies, honoraria, stock ownership or options, expert testimony, grants or patents received or pending, or royalties.

No writing assistance was utilized in the production of this manuscript.

immunological ignorance. Proc. Natl Acad. Sci. USA 96(16), 2233-2238 (1999).

10 Webb S, Morris C, Sprent J. Extrathymic tolerance of mature T cells: clonal elimination as a consequence of immunity. Cell 63(6), 1249-1256 (1990).

11 Van Elsas A, Hurwitz AA, Allison JP. Combination immunotherapy of B16 melanoma using anti-cytotoxic T lymphocyte-associated antigen 4 (CTLA- 4 ) and granulocyte/macrophage colony-stimulating factor (GM$\mathrm{CSF}$ )-producing vaccines induces rejection of subcutaneous and metastatic tumors accompanied by autoimmune depigmentation. J. Exp. Med. 190(3), 355-366 (1999).

12 Zinkernagel RM. Localization dose and time of antigens determine immune reactivity. Semin. Immunol. 12(3), 163-171; discussion 257-344 (2000).

13 Tsung K, Meko JB, Tsung YL et al. Immune response against large tumors eradicated by treatment with cyclophosphamide and IL-12. J. Immunol. 160(3), 1369-1377 (1998).

14 Le HN, Lee NC, Tsung K et al. Pre-existing tumorsensitized $\mathrm{T}$ cells are essential for eradication of established tumors by IL-12 and cyclophosphamide plus IL-12. J. Immunol. 167(12), 6765-6772 (2001).

15 Ruby CE, Montler R, Zheng R et al. IL-12 is required for anti-OX40-mediated CD4 T cell survival. J. Immunol. 180 (4), 2140-2148 (2008).

16 Valenzuela JO, Hammerbeck CD, Mescher MF. Cutting edge: Bcl-3 up-regulation by signal 3 cytokine (IL-12) prolongs survival of antigen-activated CD8 T cells. J. Immunol. 174(2), 600-604 (2005).

17 King IL, Segal BM. Cutting edge: IL-12 induces $\mathrm{CD} 4^{+} \mathrm{CD} 25^{-} \mathrm{T}$ cell activation in the presence of $\mathrm{T}$ regulatory cells. J. Immunol. 175(2), 641-645 (2005). 